\title{
Soft Chemical Method for synthesizing Intermetallic Antimonide Nanocrystals from Ternary Chalcogenide
}

Yilan Jiang", Long Yuan\#, Yanyan Xu, Jiaojiao Ma, Yu Sun, Xia Gao, Keke Huang*, Shouhua Feng

State Key Laboratory of Inorganic Synthesis and Preparative Chemistry, College of Chemistry, Jilin University, Changchun 130012, People's Republic of China

Key Laboratory of Functional Materials Physics and Chemistry of the Ministry of Education, Jilin Normal University, Changchun 130103, People's Republic of China

KEYWORDS: Antimonide, Desulfurization, Synthesis, Ternary chalcogenide, Trialkylphosphine

Containing: 3 pages, 6 Figures 


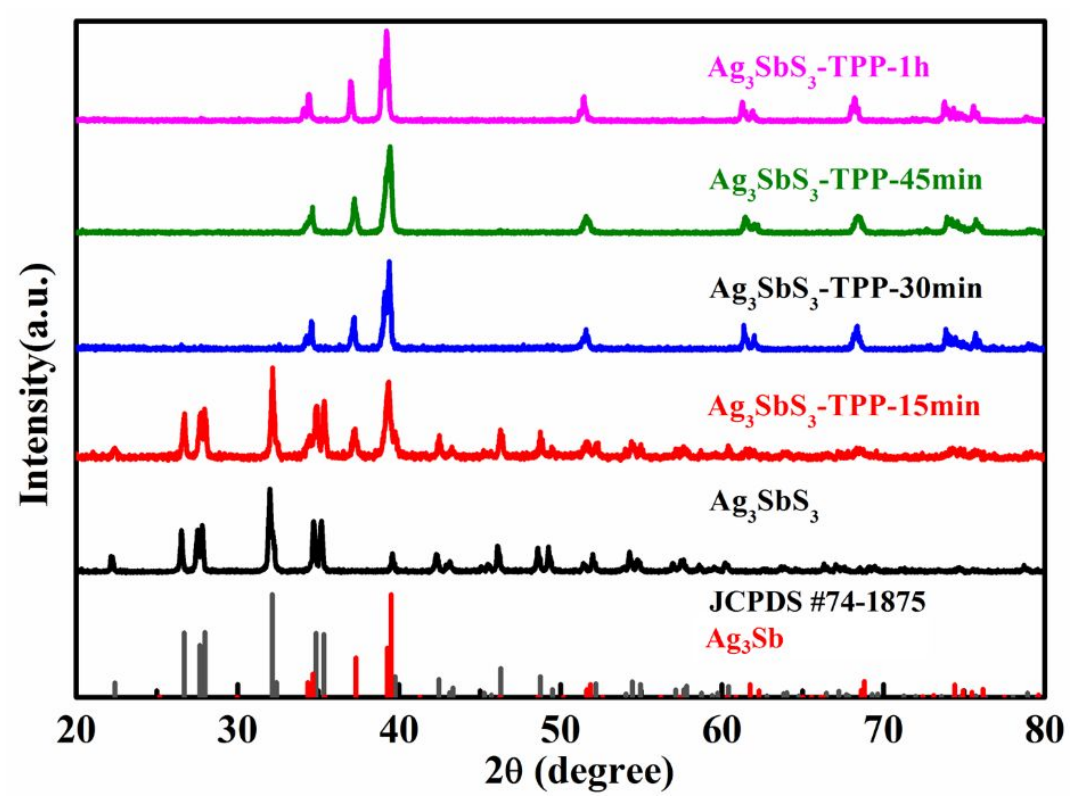

Figure S1. PXRD pattern of $\mathrm{Ag}_{3} \mathrm{SbS}_{3}, \mathrm{Ag}_{3} \mathrm{SbS}_{3}-\mathrm{TPP}-15 \mathrm{~min}, \mathrm{Ag}_{3} \mathrm{SbS}_{3}$-TPP-30 min, $\mathrm{Ag}_{3} \mathrm{SbS}_{3}$-TPP-45 min, $\mathrm{Ag}_{3} \mathrm{SbS}_{3}$-TPP-60 min.

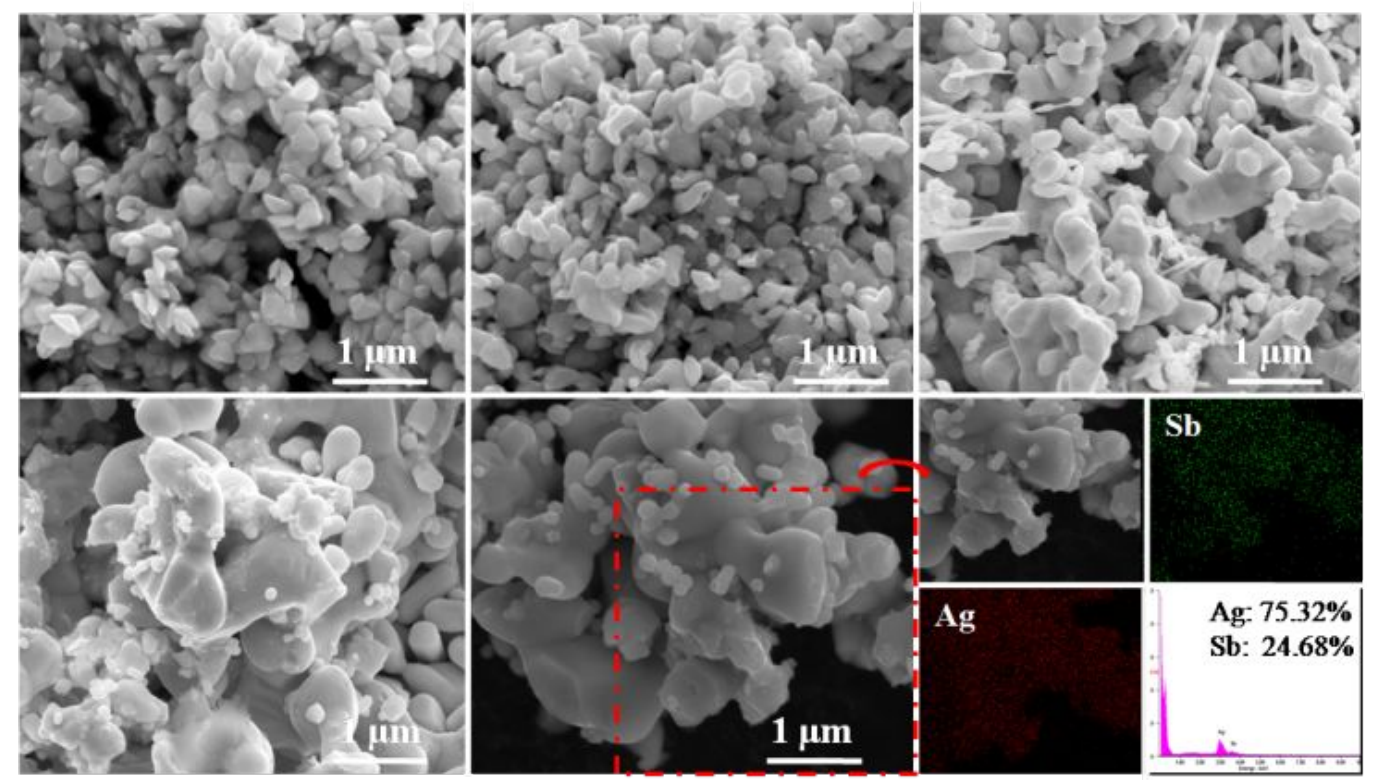

Figure S2. $\mathrm{SEM}$ images of $\mathrm{Ag}_{3} \mathrm{SbS}_{3}, \mathrm{Ag}_{3} \mathrm{SbS}_{3}-\mathrm{TPP}-15 \mathrm{~min}, \mathrm{Ag}_{3} \mathrm{SbS}_{3}-\mathrm{TPP}-30 \mathrm{~min}$,

$\mathrm{Ag}_{3} \mathrm{SbS}_{3}-\mathrm{TPP}-45 \mathrm{~min}, \mathrm{Ag}_{3} \mathrm{SbS}_{3}-\mathrm{TPP}-60 \mathrm{~min}$ and EDS mapping of $\mathrm{Ag}_{3} \mathrm{SbS}_{3}-\mathrm{TPP}-60$ $\min$ 

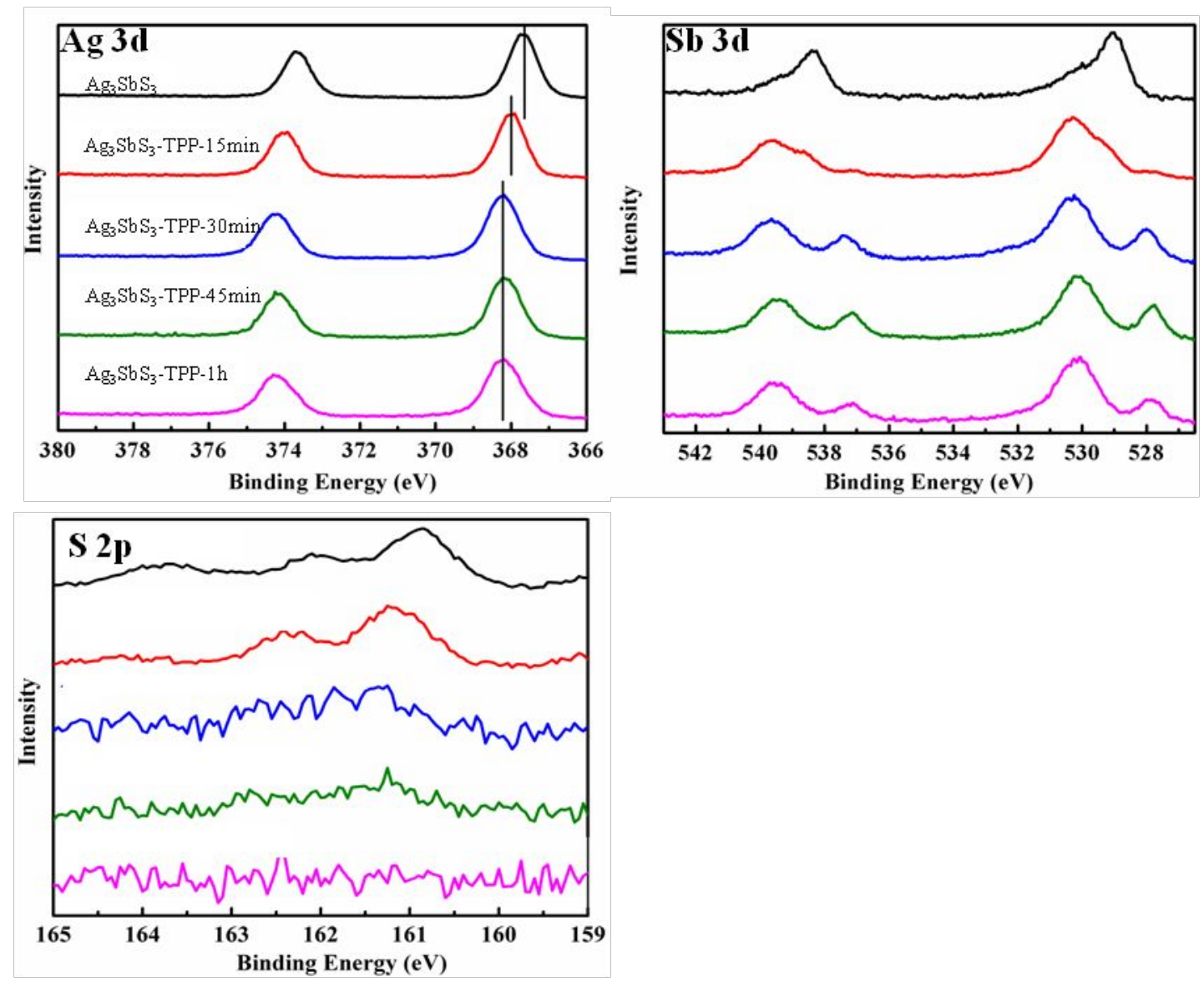

Figure S3. XPS spectrum of $\mathrm{Ag}_{3} \mathrm{SbS}_{3}, \mathrm{Ag}_{3} \mathrm{SbS}_{3}-\mathrm{TPP}-15 \mathrm{~min}, \mathrm{Ag}_{3} \mathrm{SbS}_{3}-\mathrm{TPP}-30 \mathrm{~min}$, $\mathrm{Ag}_{3} \mathrm{SbS}_{3}$-TPP-45 min, $\mathrm{Ag}_{3} \mathrm{SbS}_{3}$ - TPP-60 min and EDS mapping of $\mathrm{Ag}_{3} \mathrm{SbS}_{3}-\mathrm{TPP}-60$ $\min$
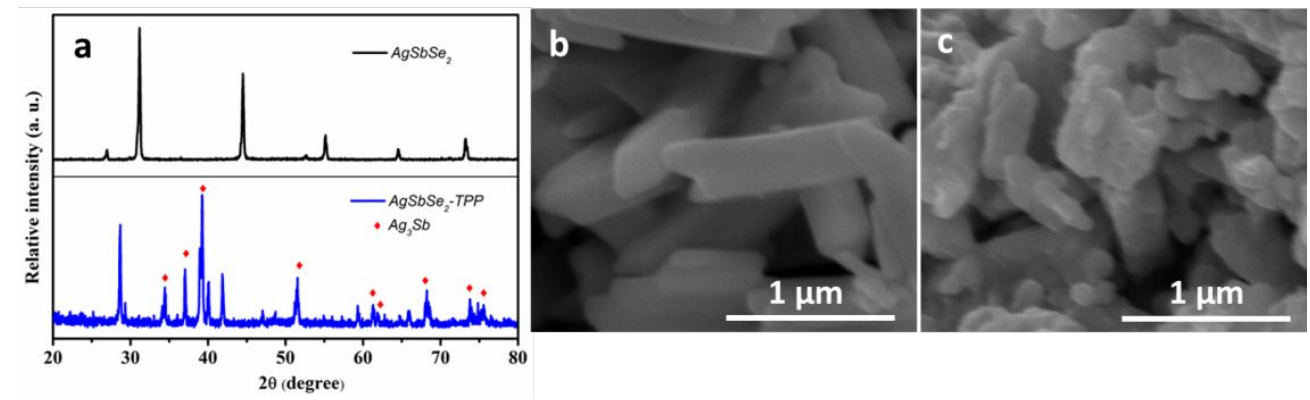

Figure S4. (a) PXRD result, (b) SEM graph of $\mathrm{AgSbSe}_{2}$ and (c) $\mathrm{SEM}$ graph of $\mathrm{Ag}_{3} \mathrm{Sb}$ nanocrystal from the deselenium of $\mathrm{AgSbSe}_{2}$. 


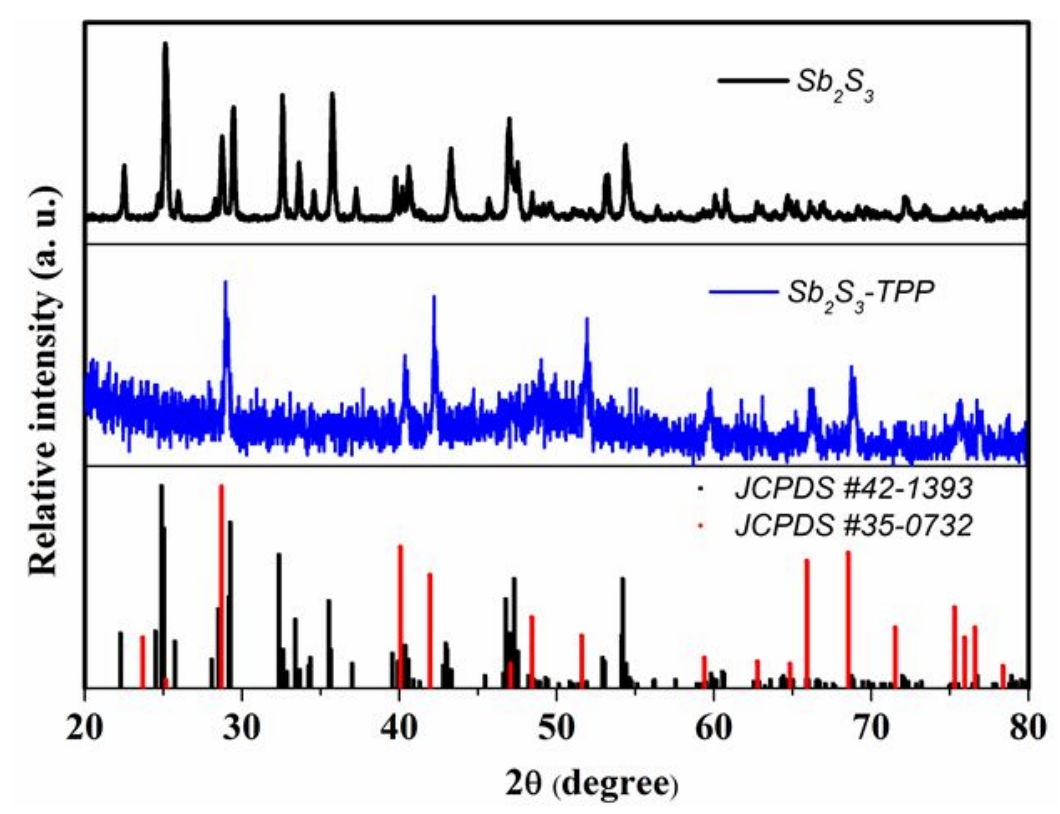

Figure S5. PXRD of $\mathrm{Sb}_{2} \mathrm{~S}_{3}$ and $\mathrm{Sb}_{2} \mathrm{~S}_{3}-\mathrm{TPP}$.
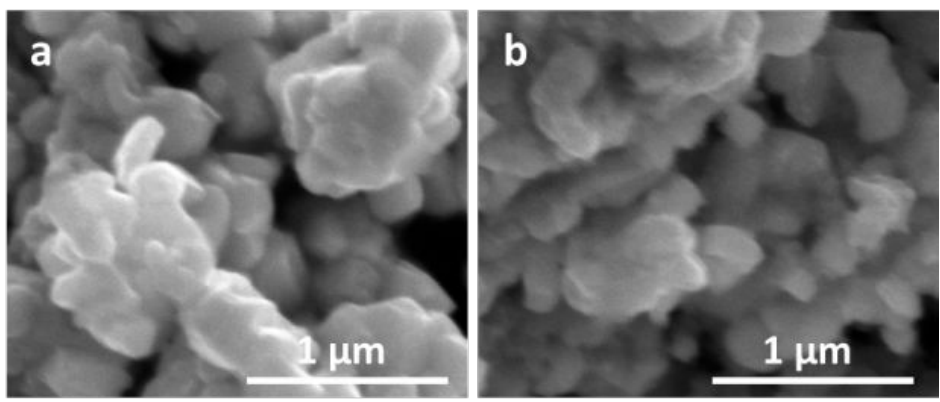

Figure S6. SEM graph of (a) $\mathrm{Sb}_{2} \mathrm{~S}_{3}$ and (b) $\mathrm{Sb}_{2} \mathrm{~S}_{3}$-TPP. 